\title{
Optics for MUSIC: a New (Sub)millimeter Camera for the Caltech Submillimeter Observatory
}

\author{
Jack Sayers $^{a, b}$, Nicole G. Czakon ${ }^{c}$, Peter K. Day ${ }^{a}$, Thomas P. Downes ${ }^{c}$, Ran P. Duan $^{c}$, \\ Jiansong Gao ${ }^{d}$, Jason Glenn ${ }^{e}$, Sunil R. Golwala ${ }^{c}$, Matt I. Hollister ${ }^{c}$, Henry G. LeDuc ${ }^{a}$, \\ Benjamin A. Mazin ${ }^{f}$, Philip R. Maloney ${ }^{e}$, Omid Noroozian ${ }^{c}$, Hien T. Nguyen ${ }^{a}$, James A. \\ Schlaerth $^{e}$, Seth Siegel ${ }^{c}$, John E. Vaillancourt ${ }^{g}$, Anastasios Vayonakis ${ }^{c}$, Philip R. Wilson ${ }^{a}$, and \\ Jonas Zmuidzinas ${ }^{c}$ \\ ${ }^{a}$ Jet Propulsion Lab, Pasadena, CA 91109 USA; \\ ${ }^{b}$ NASA Postdoctoral Program Scholar; \\ ${ }^{c}$ California Institute of Technology, Pasadena, CA 91125 USA; \\ ${ }^{d}$ National Institute of Standards and Technology, Boulder, CO 80305 USA; \\ ${ }^{e}$ Center for Astrophysics \& Space Astronomy, University of Colorado, Boulder, CO 80309 USA; \\ ${ }^{f}$ University of California, Santa Barbara, CA 93106 USA; \\ ${ }^{g}$ Stratospheric Observatory for Infrared Astronomy, Moffett Field, CA 94035 USA
}

\begin{abstract}
We will present the design and implementation, along with calculations and some measurements of the performance, of the room-temperature and cryogenic optics for MUSIC, a new (sub)millimeter camera we are developing for the Caltech Submm Observatory (CSO). The design consists of two focusing elements in addition to the CSO primary and secondary mirrors: a warm off-axis elliptical mirror and a cryogenic (4K) lens. These optics will provide a 14 arcmin field of view that is diffraction limited in all four of the MUSIC observing bands $(2.00,1.33,1.02$, and $0.86 \mathrm{~mm})$. A cold $(4 \mathrm{~K})$ Lyot stop will be used to define the primary mirror illumination, which will be maximized while keeping spillover at the sub $1 \%$ level. The MUSIC focal plane will be populated with broadband phased antenna arrays that efficiently couple to factor of $\simeq 3$ in bandwidth, ${ }^{1,2}$ and each pixel on the focal plane will be read out via a set of four lumped element filters that define the MUSIC observing bands (i.e., each pixel on the focal plane simultaneously observes in all four bands). Finally, a series of dielectric and metal-mesh low pass filters have been implemented to reduce the optical power load on the MUSIC cryogenic stages to a quasi-negligible level while maintaining good transmission in-band.
\end{abstract}

Keywords: MUSIC, MKID, Relay Optics, Caltech Submillimeter Observatory

\section{INTRODUCTION}

MUSIC (the Multicolor Submillimeter Inductance Camera) is a new camera that will soon begin collecting data at the $\mathrm{CSO}_{,}^{3,4}$ replacing the existing mm-wave imaging camera Bolocam. ${ }^{5}$ The MUSIC focal plane will be populated with $576(24 \times 24)$ phased antenna arrays, ${ }^{1}$ which will be coupled to a new type of pair-breaking detector, a microwave kinetic inductance detector (MKID). ${ }^{6}$ MUSIC will have a 14 arcmin field of view (FOV), which provides a detector spacing of $\simeq(\mathrm{f} / \#) \lambda$. Lumped-element band defining filters will be placed between the phased antenna arrays and the MKIDs ${ }^{7}$ and four observing bands $(2.00,1.33,1.02$, and $0.86 \mathrm{~mm})$ will be defined for each pixel (i.e., each pixel will be coupled to four MKIDs). Based on MUSIC's spectral coverage, it is an ideal instrument for observing objects such as high-redshift dusty submm galaxies ${ }^{8}$ and massive galaxy clusters via the Sunyaev-Zel'dovich effect (SZE). ${ }^{9}$

In this paper we will present the details of the MUSIC optical design. The design naturally splits into two separate systems: one that defines the point-spread function, FOV, etc. (i.e., elements that reflect, refract, or define apertures) and one that shields the focal plane from unwanted optical power and defines the observing bands (i.e., elements that filter the incoming radiation).

Further author information: (Send correspondence to Jack Sayers)

E-mail: jack@caltech.edu.edu, Telephone: 16263952892

Millimeter, Submillimeter, and Far-Infrared Detectors and Instrumentation for Astronomy V, edited by Wayne S. Holland, Jonas Zmuidzinas, Proc. of SPIE Vol. 7741, 77410W (c) 2010 SPIE - CCC code: 0277-786X/10/\$18 - doi: 10.1117/12.857324

Proc. of SPIE Vol. 7741 77410W-1 


\section{REFLECTIVE, REFRACTIVE, AND APERTURE DEFINING OPTICAL COMPONENTS}

\subsection{OVERVIEW}

The Cassegrain telescope at the CSO consists of a $10.4 \mathrm{~m}$ diameter primary mirror and $\mathrm{a} \simeq 50 \mathrm{~cm}$ diameter secondary mirror. Due to the focal ratio from the secondary mirror $(F \simeq 12.5)$, and corresponding platescale at the Cassegrain focus $(\simeq 1.5 \mathrm{arcsec} / \mathrm{mm})$, reimaging relay optics are required to couple a large FOV camera to the telescope. Currently, the 8 arcmin FOV provided by the SuZIE II relay optics is the largest at the CSO. ${ }^{5}$ With MUSIC's focal plane, an 8 arcmin FOV would provide a pixel spacing of $\simeq 0.4$ (f/\#) $\lambda$ at $2.00 \mathrm{~mm}$ and $\simeq 0.9(\mathrm{f} / \#) \lambda$ at $0.85 \mathrm{~mm}$; this approximately Nyquist spacing would be efficient for mapping compact sources with MUSIC, ${ }^{10}$ but the sensitivity per-pixel (and hence the total camera mapping speed given our fixed number of detectors) would be maximized with a pixel spacing of $\simeq 2(\mathrm{f} / \#) \lambda .{ }^{11}$ Since MUSIC will be used primarily for large surveys, our goal is to maximize the mapping speed of the camera, which means we would like much larger pixel spacings than can be achieved with the 8 arcmin FOV of the SuZIE II relay optics. Therefore, we are fabricating new relay optics to provide the largest FOV possible at the CSO.

The FOV limiting aperture at the CSO is a tubular opening in the hexplate that forms the base of the support structure for the primary mirror and has a diameter of $\simeq 60 \mathrm{~cm}$; since this aperture is close to the Cassegrain focus of the telescope it truncates the FOV in a relatively clean manner. Geometrically this aperture corresponds to a diameter of $\simeq 18$ arcmin, but MUSIC's quasi-optical mm-wave beams will be noticeably vignetted $(\gtrsim 1 \%)$ for FOVs larger than $\simeq 15$ arcmin. Therefore, we have chosen to illuminate MUSIC's square focal plane such that each edge corresponds to 14 arcmin; this provides the approximately optimal combination of unvignetted detector yield and per-pixel sensitivity.

\subsection{FOCAL PLANE GEOMETRY}

MUSIC's focal plane will be populated with 576 phased antenna arrays, ${ }^{1}$ or pixels, layed out in a square with $24 \times 24$ pixels; each antenna array will be $4.2 \mathrm{~mm}$ square, and the center-to-center spacing will be $\simeq 5 \mathrm{~mm}$. Overall, the focal plane will be approximately $135 \mathrm{~mm}$ square, which means the platescale will need to be $\simeq 6.25 \mathrm{arcsec} / \mathrm{mm}$. For the $10.4 \mathrm{~m}$ primary mirror at the CSO, this platescale corresponds to a focal ratio of $\simeq 3.2^{*}$, which is well matched to our phased antenna arrays that have outgoing time-reverse focal ratios of $\simeq 2-4$ for our observing bands.

\subsection{OPTICAL DESIGN}

The simplest way to reimage a Cassegrain system is with two folding flat mirrors and a single off-axis elliptical mirror, so that basic layout was the starting point for a new relay optics design. Geometrically, the Cassegrain focus is placed at one foci of the ellipse, and the ellipse reimages the Cassegrain focus at the other foci, at least in the traditional design. However, Serabyn ${ }^{12}$ found that the unaberrated FOV could be enhanced by using a modified ellipse, where the foci is placed at a location given by $2 f$, where $f$ is the distance from the mirror surface to the Cassegrain focus. We tried varying the foci location between $1.5 f$ and $2.5 f$ and found that the aberrations are indeed minimized when the foci is placed at $2 f$, so we fixed the location of the ellipse foci at this value in all of our designs.

The relay optics and MUSIC cryostat will be mounted above the alidade platform at the CSO, which forces strict limits on the size of the optics. First, there is only enough space left-right (along the azimuth direction) for mirrors that are $\lesssim 100 \mathrm{~cm}$ in width. This means that our elliptical mirror needs to be placed $\lesssim 200 \mathrm{~cm}$ from the Cassegrain focus of the telescope, since the extreme rays for a 14 arcmin FOV will diverge by more than $100 \mathrm{~cm}$ beyond that location. Second, the primary image formed by the elliptical mirror needs to be inside the cryostat so that the Lyot stop can be placed at a cold $(\simeq 4 \mathrm{~K})$ surface $^{\dagger}$. Consequently, the primary image will need to be $\gtrsim 100 \mathrm{~cm}$ from the elliptical mirror to prevent the cryostat from blocking the optical path from the preceding

\footnotetext{
${ }^{*}$ The Lyot stop effectively makes the primary mirror smaller, which means the actual f/\# at the focal plane will be slightly larger than 3.2 .

${ }^{\dagger}$ Our pixels efficiently couple to a large amount of area outside the Lyot stop. Therefore, by placing the Lyot stop on a cold surface we can significantly reduce the amount of stray light power that is absorbed by the detectors.
} 

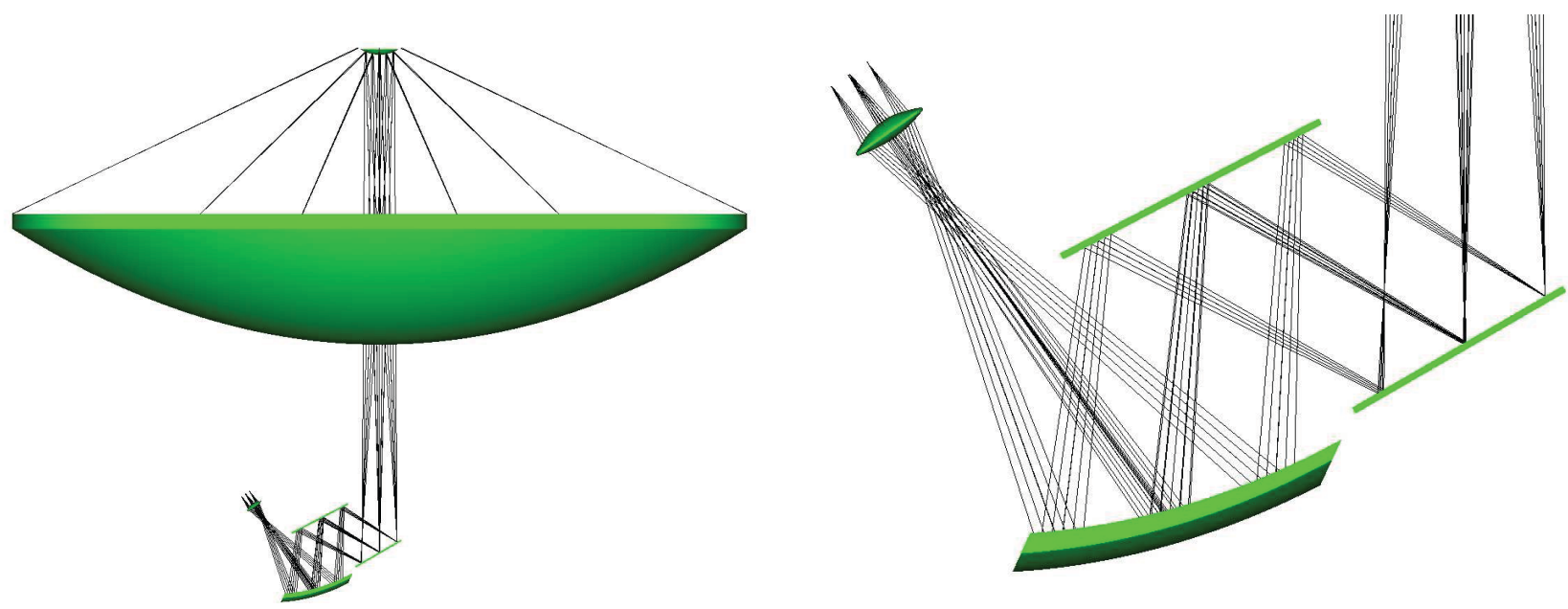

Figure 1. Left: overall view of the optical system including the $10.4 \mathrm{~m}$ primary mirror of the CSO. Right: closer view of the MUSIC relay optics, including the two folding flat mirrors, off-axis elliptical mirror, and cold reimaging lens. Note that the ray bundles represent field locations of $(+7,0),(-7,0),(0,+7),(0,-7)$ arcmin $($ az,el).

flat mirror. Note that to satisfy this requirement the focal ratio produced by the elliptical mirror will need to be $\gtrsim 10$. Since we would like to feed the MUSIC focal plane with a focal ratio of $\simeq 3$, this means we will require a reimaging lens behind the Lyot stop. Finally, the distance from the elliptical mirror to the primary image must be less than $\simeq 150 \mathrm{~cm}$ to maintain adequate clearance between the cryostat and the telescope backing structure.

Given the above constraints, we iteratively converged to an optimal design as follows: 1) pick a geometry for the two flat mirrors and single off-axis elliptical mirror, 2) determine the geometry of a cold lens that will produce the correct focal ratio for our pixels while minimizing aberrations and making the system as telecentric as possible, 3) perform time-reverse physical optics calculations using Zemax to determine the mirror sizes required so that $\gtrsim 99 \%$ of the power propagates through the relay optics, 4) confer with the design engineer to determine if a structure can be fabricated to mount the mirrors and cryostat. Note that while picking a geometry in step 1 we tried to minimize the fold angle at the elliptical mirror, since larger fold angles move the mirror further off-axis and produce worse aberrations.

For our final design the first folding mirror has dimensions of $70 \times 75 \mathrm{~cm}$ (az and el), a fold angle of $60^{\circ}$, and is located $5 \mathrm{~cm}$ in front of the telescope Cassegrain focus. The second flat is located $95 \mathrm{~cm}$ from the first flat mirror, and is $80 \times 90 \mathrm{~cm}$ with a fold angle of $65^{\circ}$; the elliptical mirror is located $105 \mathrm{~cm}$ from the second flat mirror, has a fold angle of $40^{\circ}$, and is $94 \times 97 \mathrm{~cm}$. Finally, the focal ratio coming from the elliptical mirror is $F \simeq 16$; an image of the primary mirror is formed $124 \mathrm{~cm}$ from its surface. A view of this new relay optics design is given in Figure 1. Note that the fold angles of the flat mirrors were chosen so that the cryostat will be at an angle of $35^{\circ}$ from the optical axis of the telescope. Since most of our observations will be at elevation angles of $40-70$ degrees, the cryostat will be vertical at the average elevation angle of our observations, $55^{\circ}$. The reason we would like the cryostat to be close to vertical for all our observations is because the cryomechanical cooler performance degrades when it is tilted. ${ }^{13}$

As mentioned above, the lens geometry was chosen not only to minimize aberrations over the MUSIC FOV, but also to make the system as telecentric as possible. While optimizing the properties of the lens we found that a the total level of aberrations is reasonably constant over a wide range of lens shapes/positions. However, the time-reverse illumination pattern on the primary mirror quickly becomes sub-optimal when the system becomes less telecentric. Therefore, telecentricity was the primary characteristic in determining the optimum shape and position of the lens. To this end, we found that decentering the lens and focal plane by $5 \mathrm{~mm}$ along the elevation direction significantly improves the telecentricity of the system without noticeably changing the level of aberrations. The final lens design, using ultra-high molecular weight polyethylene (UHMWPE), has a thickness of $63.5 \mathrm{~mm}$ and a diameter of $250 \mathrm{~mm}$. The radius of curvature of the lens is slightly different on each face, and is $\simeq 250 \mathrm{~mm}$. 


\begin{tabular}{|c|cccc|}
\hline Lyot stop $\varnothing$ & M2 spillover & M1 spillover & PSF FWHM & $N E P_{\gamma}(\mathrm{mJy} / \sqrt{\mathrm{Hz}})$ \\
\hline $7.8 \mathrm{~m}$ & $0.1 \%$ & $0.0 \%$ & 51 arcsec & 23 \\
$8.7 \mathrm{~m}$ & $0.7 \%$ & $0.2 \%$ & 47 arcsec & 17 \\
$9.0 \mathrm{~m}$ & $1.1 \%$ & $0.5 \%$ & $45 \operatorname{arcsec}$ & 16 \\
$9.6 \mathrm{~m}$ & $1.7 \%$ & $0.9 \%$ & $44 \operatorname{arcsec}$ & 15 \\
$10.4 \mathrm{~m}$ & $3.7 \%$ & $4.4 \%$ & $42 \operatorname{arcsec}$ & 14 \\
\hline
\end{tabular}

Table 1. Spillover fraction at the secondary mirror (M2) and primary mirror (M1), full-width at half-maximum for the farfield PSF, and estimated point-source sensitivity for a range of effective Lyot stop diameters. Values for the $2.00 \mathrm{~mm}$ band are shown because the mirror spillover is largest for this band, however the relative increase in spillover and improvement in point-source sensitivity is similar for the other three bands. An effective Lyot stop diameter of $9.0 \mathrm{~m}$ will be used for MUSIC.

Finally, our optical design includes a Lyot stop at the primary image located between the cold reimaging lens and the elliptical mirror. A significant fraction, roughly $50 \%$, of the time-reverse beam for our $\simeq(\mathrm{f} / \#) \lambda$ pixels is truncated at the Lyot stop (see Table 2); to limit the amount of stray light power absorbed by the detectors the Lyot stop will be maintained at $\simeq 4 \mathrm{~K}$. The size of the Lyot stop was chosen to optimally balance the tradeoff between optical efficiency/primary illumination and mirror spillover. By illuminating more of the primary, which will narrow the far-field point-spread function (PSF), we can significantly improve MUSIC's mapping speed for the point-like galaxies and relatively compact galaxy clusters we will be observing ${ }^{\ddagger}$. However, this narrowing of the PSF needs to be balanced against the increased optical load and potential systematics produced by increased mirror spillover. Since the sky is relatively cold at $\mathrm{mm}$ wavelengths the sensitivity penalty for spillover at the secondary mirror is benign, but spillover at the primary mirror will couple to warm surfaces which may or may not have fluctuating temperatures. We have used Zemax to predict the amount of primary/secondary mirror spillover for various Lyot stop diameters, and we have calculated a corresponding point-source sensitivity for each case. ${ }^{14}$ Based on this analysis, we have chosen a Lyot stop with a diameter of $69.4 \mathrm{~mm}(9.0 \mathrm{~m}$ at the primary mirror); at larger diameters the improvement in point-source sensitivity is milder while the amount of spillover increases significantly. See Table 1. Note that the primary mirror spillover is predicted to be less than $1 \%$ for all four MUSIC observing bands.

\subsection{MECHANICAL DESIGN}

We have designed, and are currently fabricating, a single optics box that contains the two flat mirrors and the off-axis elliptical mirror; this box mounts to the telescope backing structure and provides a mounting point for the camera cryostat. An overall view of the design is given in Figure 2. After iterating to a mechanical design that was compatible with our optical design (see Section 2.3), our only additional requirement was that the structure be rigid enough that it will not noticeably affect our optical performance under the stresses it will experience at the telescope (e.g., from gravity and telescope scanning). The intrinsic pointing uncertainty of the CSO is $2-3$ arcsec, which means that the pointing uncertainty induced by deformations of the optics box needs to be small compared to this value. Additionally, changes in the optical efficiency (strehl ratio) need to be small compared to our inherent flux calibration uncertainty, which is $\simeq 5 \%$.

Using finite element analysis, we have calculated the amount of translation, tilt, and deformation that can be expected for each optical element in our system. Zemax has been used to determine the associated optical performance degradations resulting from these translations and deformations. In all cases the change in strehl ratio is negligible compared to our expected flux calibration uncertainty, and the pointing errors are small compared to the intrinsic pointing uncertainty of the CSO. A summary is given in Table 3. Note that our individual raster scans of a source will be $\lesssim 5 \mathrm{deg}^{\S}$, which means the maximum differential force due to gravity on any element in our system will be $g / 12$. We will ignore the acceleration of the telescope during a scan since it will be negligible compared to the differential gravitational acceleration .

\footnotetext{
${ }^{\ddagger}$ Additionally, source confusion will be a major issue for deep surveys for submm galaxies with MUSIC; a narrower PSF will reduce the amount of confusion for a given source flux.

${ }^{\S}$ It is possible to accurately fit for pointing drifts between scans as a source moves across the sky, ${ }^{15}$ therefore it is only
} 


\begin{tabular}{|c|cccccc|}
\hline band/location & Lyot stop & filters/window & relay mirrors & primary supports & secondary & primary \\
\hline $2.00 \mathrm{~mm}(0,0)$ & $72.1 \%$ & $0.1 \%$ & $0.4 \%$ & $0.1 \%$ & $1.1 \%$ & $0.5 \%$ \\
$2.00 \mathrm{~mm}(0,+7)$ & $73.1 \%$ & $0.2 \%$ & $2.1 \%$ & $2.8 \%$ & $4.3 \%$ & $0.8 \%$ \\
$2.00 \mathrm{~mm}(+7,0)$ & $73.1 \%$ & $0.2 \%$ & $2.0 \%$ & $0.9 \%$ & $1.5 \%$ & $0.5 \%$ \\
\hline $1.33 \mathrm{~mm}(0,0)$ & $49.0 \%$ & $0.0 \%$ & $0.1 \%$ & $0.1 \%$ & $0.3 \%$ & $0.1 \%$ \\
$1.33 \mathrm{~mm}(0,+7)$ & $49.9 \%$ & $0.1 \%$ & $1.1 \%$ & $1.5 \%$ & $3.4 \%$ & $0.3 \%$ \\
$1.33 \mathrm{~mm}(+7,0)$ & $50.4 \%$ & $0.1 \%$ & $0.9 \%$ & $0.5 \%$ & $0.7 \%$ & $0.2 \%$ \\
\hline $1.02 \mathrm{~mm}(0,0)$ & $32.4 \%$ & $0.0 \%$ & $0.1 \%$ & $0.0 \%$ & $0.2 \%$ & $0.0 \%$ \\
$1.02 \mathrm{~mm}(0,+7)$ & $32.7 \%$ & $0.0 \%$ & $0.6 \%$ & $0.7 \%$ & $2.0 \%$ & $0.1 \%$ \\
$1.02 \mathrm{~mm}(+7,0)$ & $33.7 \%$ & $0.0 \%$ & $0.5 \%$ & $0.3 \%$ & $0.3 \%$ & $0.0 \%$ \\
\hline $0.86 \mathrm{~mm}(0,0)$ & $23.8 \%$ & $0.0 \%$ & $0.1 \%$ & $0.0 \%$ & $0.1 \%$ & $0.0 \%$ \\
$0.86 \mathrm{~mm}(0,+7)$ & $24.1 \%$ & $0.0 \%$ & $0.3 \%$ & $0.4 \%$ & $1.4 \%$ & $0.0 \%$ \\
$0.86 \mathrm{~mm}(+7,0)$ & $25.1 \%$ & $0.0 \%$ & $0.3 \%$ & $0.2 \%$ & $0.1 \%$ & $0.0 \%$ \\
\hline
\end{tabular}

Table 2. Spillover fraction for each surface, or set of surfaces, in the optical system. The spillover is calculated for the Lyot stop, the filters and window of the cryostat, the relay mirrors (two flat mirrors and one off-axis elliptical mirror), the opening in the support structure for the CSO primary mirror, the secondary mirror, and the primary mirror. For each observing band we have calculated the spillover for a pixel at the center of the focal plane $(0,0$ arcmin $)$, one at the extreme edge of the focal plane in the elevation direction $(0,+7$ arcmin $)$, and one at the extreme edge of the focal plane in the azimuth direction $(+7,0$ arcmin).

We have also used Zemax to determine the manufacturing surface tolerances for all of the MUSIC mirrors. The elliptical mirror will be able to translate perpendicular to the optical axis by \pm 0.5 inches to account for small misalignments (misalignments perpendicular to the optical axis will not affect the performance of the flat mirrors). Small misalignments of the mirrors along the optical axis can be corrected using the adjustable secondary mirror at the CSO. Additionally, the optics box design will allow us to adjust each mirror by $\pm 0.4 \mathrm{deg}$, so we can easily compensate for any imperfection in the mirror surfaces that appears as a slight tilt. However, there will also be higher-order imperfections in the surfaces that will degrade the optical performance of the system; we have modeled these deformations as an effective change in the radius of curvature of the mirror. In some cases, this degradation can be partially compensated for by adjusting the position of the CSO secondary mirror, but there is still a reduction in the strehl ratio of the system. We have specified a maximum surface deviation of $0.05 \mathrm{~mm}$ (0.002 inches) for each mirror surface; this deviation is caused primarily by internal material stress and will appear as a large scale deformation of the mirror. This tolerance is not difficult to achieve for our $\simeq 100 \mathrm{~cm}$ mirrors, and such deviations reduce the strehl ratio of the system by less than $0.02 \%, 0.10 \%$, and $0.08 \%$

the pointing drift within a scan that we are concerned with.

"The maximum acceleration of the $\mathrm{CSO}$ is $\simeq 1$ arcmin-s ${ }^{-2}$, which corresponds to $\simeq g / 5000$.
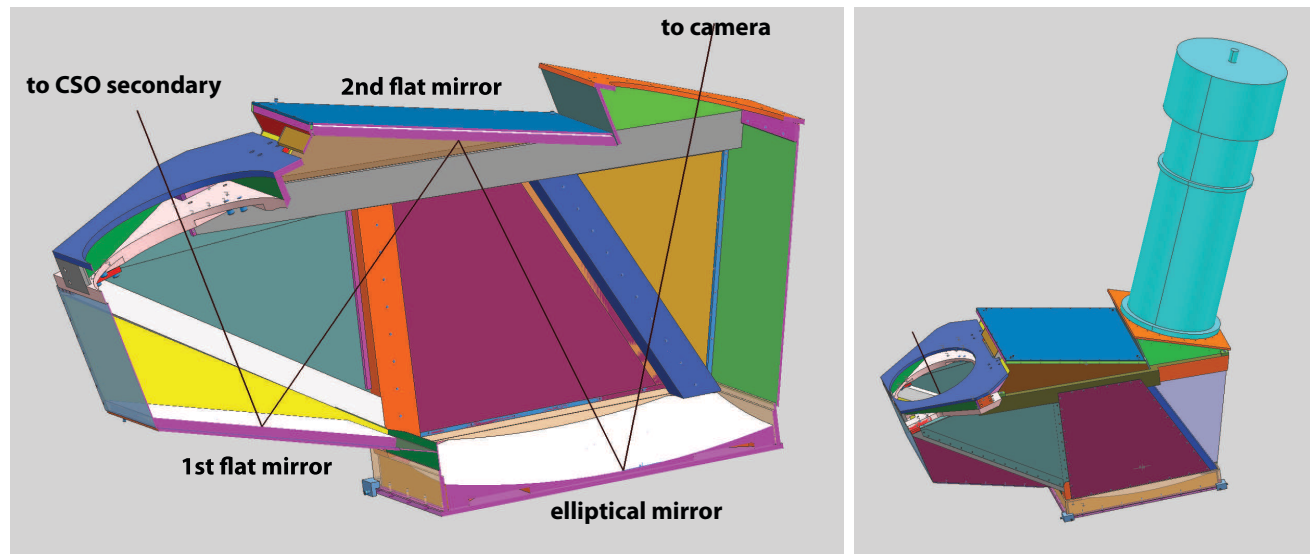

Figure 2. Left: cross section of the relay optics box. Right: isometric view of the optics box and camera cryostat. 


\begin{tabular}{|c|cc|cc|cc|}
\hline surface & translation & $\Delta$ strehl & tilt & pointing err. & surface deformation & $\Delta$ strehl \\
\hline M3 (first flat) & $20 \mu \mathrm{m}$ & $0.01 \%$ & $0.3 \mu \mathrm{m}$ & $0.1 \operatorname{arcsec}$ & $R_{\text {curv }}=2 \times 10^{8} \mathrm{~mm}$ & $<0.01 \%$ \\
M4 (second flat) & $30 \mu \mathrm{m}$ & $0.01 \%$ & $0.7 \mu \mathrm{m}$ & $0.2 \operatorname{arcsec}$ & $R_{\text {curv }}=1 \times 10^{8} \mathrm{~mm}$ & $0.01 \%$ \\
M5 (elliptical) & $30 \mu \mathrm{m}$ & $0.01 \%$ & $2.4 \mu \mathrm{m}$ & $0.5 \operatorname{arcsec}$ & $R_{\text {curv }}=5 \times 10^{7} \mathrm{~mm}$ & $0.02 \%$ \\
focal plane & $80 \mu \mathrm{m}$ & $0.11 \%$ & N/A & $0.5 \operatorname{arcsec}$ & N/A & N/A \\
\hline
\end{tabular}

Table 3. The maximum changes in the optical performance of MUSIC for translations, tilts, and surface deformations of the optical elements during a $5 \mathrm{deg}$ raster scan of the CSO in the elevation direction. These changes in the optical properties are caused by differences in the gravitational force on the MUSIC optics during the scan. For the three mirrors, translations and surface deformations cause changes in the strehl ratio and tilts result in pointing errors. For the focal plane, translations along the optical axis, tilts, and surface deformations cause changes in the strehl ratio and translations perpendicular to the optical axis result in pointing errors; therefore we have lumped all of the motions of the focal plane into a single "translation" value, and given the associated change in strehl ratio and pointing. Note that the focal plane translations are due to roughly equal contributions from the optics box that the cryostat mounts to along with support structures within the cryostat. In all cases the change in the strehl ratio is negligible compared to the $\simeq 5 \%$ flux calibration error we expect for MUSIC data, and the pointing errors are small compared to the $2-3$ arcsec uncertainty inherent to the CSO.

for the first flat, the second flat, and the elliptical mirror. The surface finish of each mirror will be $0.004 \mathrm{~mm}$ (0.0001 inches), limited by the minimum scallop height between cuts that is cost-feasible for the fabrication of the elliptical mirror. Such surface imperfections will produce losses of $\lesssim 0.3 \%$.

Additionally, we are currently fabricating all of the cryogenic optical components. In addition to the lens and Lyot stop, we will also have several baffles with a temperature of $\simeq 4 \mathrm{~K}$ to reduce the amount of stray light power absorbed by the detectors. Although the phased antenna arrays produce a fairly narrow time-reverse beam, a significant amount of the beam is terminated at the Lyot stop; there is also non-zero response at large angles outside the main beam. Starting from the focal plane, there is a cylindrical baffle with the same diameter as the cold lens that extends out from the focal plane $18 \mathrm{~cm}$. A second cylindrical baffle, with slightly larger diameter, extends towards the focal plane from the lens and overlaps the first baffle by $8 \mathrm{~cm}$. A third cylindrical baffle, again with a diameter equal to the cold lens, extends from the opposite side of the lens a distance of $11 \mathrm{~cm}$. The Lyot stop is then cut out from the end plate of a cylinder that extends well past the preceding baffles. See Figure 3. All of these surfaces will be covered with carbon-loaded stycast, ${ }^{16}$ trowled to have a v-shaped profile. Consequently, all of the baffle surfaces will be extremely emissive, ensuring that approximately all of the stray light absorbed by the detectors will be from $4 \mathrm{~K}$ surfaces.

Finally, we have already designed, built, and installed a new upper relay assembly for the CSO. This assembly contains two computer-controlled movable mirror mounts, one for each Nasmyth focus of the telescope, along with mechanical support and mounting points for relay optics at the Cassegrain focus, such as MUSIC. The previous assembly had to be replaced because it only provided $\mathrm{a} \simeq 33 \mathrm{~cm}$ clear aperture for the Cassegrain focus,
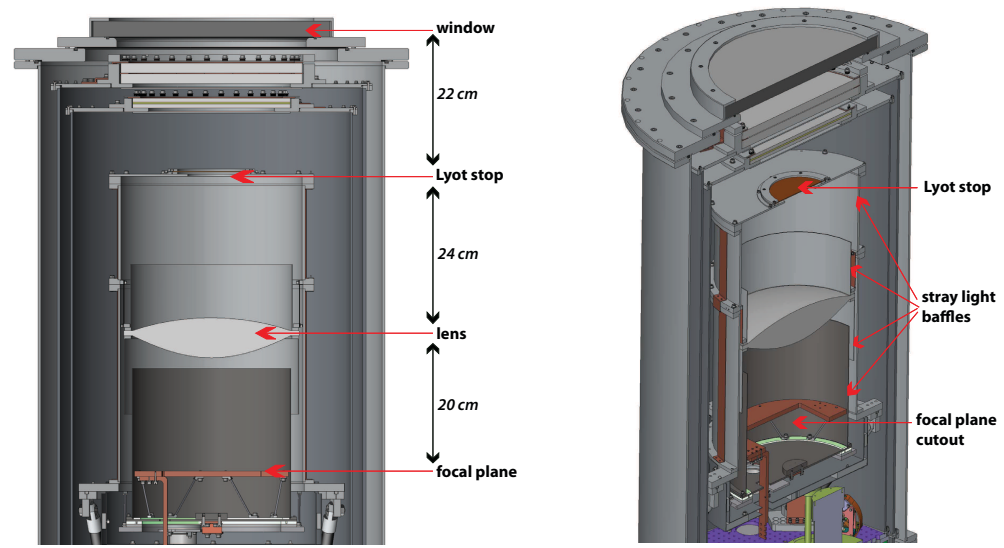

Figure 3. Left: cross section of the camera cryostat. Right: isometric view of the same cross section. 

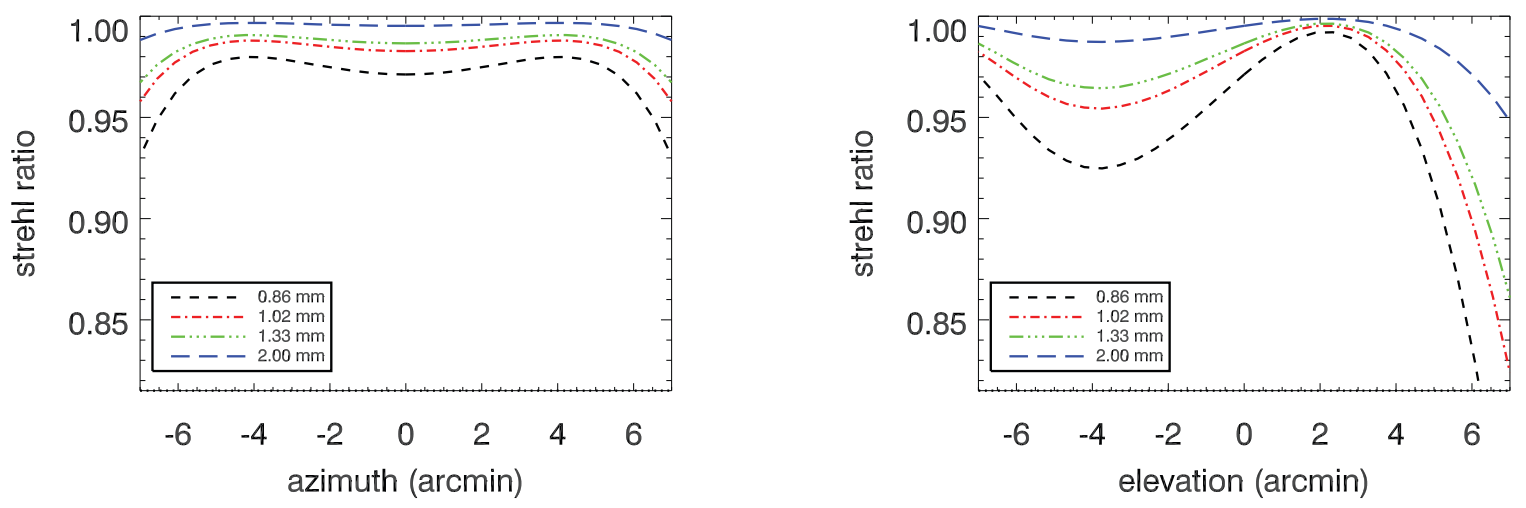

Figure 4. Strehl ratio over the MUSIC FOV for cuts in azimuth (left) and elevation (right) for all four MUSIC observing bands.

which would have limited the FOV to $\lesssim 10$ arcmin. The new assembly has a $60 \mathrm{~cm}$ clear aperture to ensure that the opening in the immutable telescope backing structure is the FOV limiting aperture in the system.

\subsection{MEASURED AND CALCULATED PERFORMANCE}

Since the MUSIC camera is still in development, and the optics box is currently being fabricated, we have not measured the end-to-end performance of the full optical system. However, we have measured the performance of some of the sub-components in the system, and we have estimated the performance via simulation for the entire system using Zemax.

As mentioned in Section 2.3, one of the drivers of our optical design was maximizing the strehl ratio over the entire FOV. This optimization was performed using Zemax, and our final design produces strehl ratios above the diffraction limit for all four MUSIC bands over the entire FOV, with the exception of the extreme edge of the FOV for the $0.86 \mathrm{~mm}$ band. See Figure 4.

Additionally, we have used Zemax to calculate the far-field PSF for pixels in all four bands at the center and edges of the FOV. The predicted FWHMs of the PSFs for pixels at the center of the focal plane are 45, 31, 25, and $22 \operatorname{arcsec}$ for the 2.00,1.33, 1.02, and $0.86 \mathrm{~mm}$ observing bands. Pixels at the edge of the focal plane are predicted to have FHWMs that are slightly larger $(\simeq 2-3 \%)$. See Figure 5 . We have recently completed an observing run with a test focal plane using the existing SuZIE II relay optics box; ${ }^{17}$ the pixels in this focal plane are identical to what will be in the final camera, but we did not include the $2.00 \mathrm{~mm}$ band in this test device. Additionally, the Lyot stop used for this test run has an effective diameter of $7.8 \mathrm{~m}$, as opposed to $9.0 \mathrm{~m}$ for the final camera. As a result, the PSFs of the test camera are predicted to be 36,28 , and 24 arcsec for the 1.33, 1.02 , and $0.86 \mathrm{~mm}$ bands. The measured FWHMs, from observations of Uranus, are $35.7 \pm 1.1,29.5 \pm 1.3$, and $25.7 \pm 1.0$ arcsec for the three observing bands, close to the predicted values.

\section{FILTERING AND BAND-DEFINING COMPONENTS}

\subsection{OVERVIEW}

The detectors used in MUSIC require an operating temperature of $\simeq 250 \mathrm{mK}$ to optimize their sensitivity, so the camera focal plane is cooled inside a cryostat using a Cryomech PT- 415 and a Chase He-10 sorption fridge. ${ }^{13,18,19}$ We require a large $(\simeq 300 \mathrm{~mm}$ diameter $)$ window in this cryostat; the optical power that passes through this window produces a noticeable load on the first stage of the PT-415, and significantly exceeds the cooling power of the second stage of the PT- 415 and the He-10 fridge. Most of this optical load is due to photons at wavelengths much shorter than our observing bands, and we have designed a filtering scheme to absorb/reflect this shortwavelength radiation without significantly decreasing the in-band transmission (the transmission is $\gtrsim 85 \%$ in all 

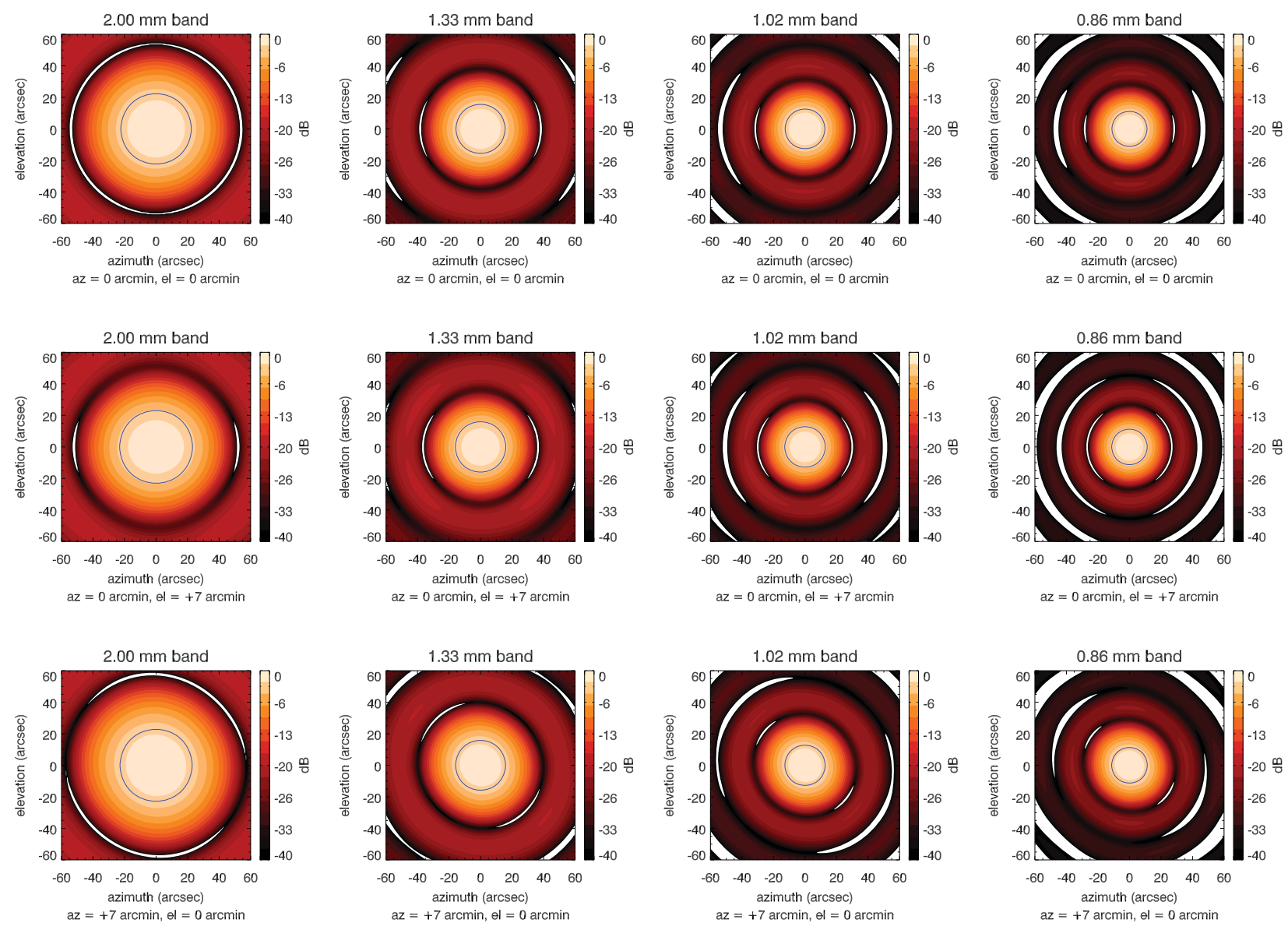

Figure 5. Far field PSF for pixels at the center (top) and edges (center, bottom) of the FOV for the four MUSIC observing bands (left to right). The blue circle indicates the FWHM.

four bands). The resulting optical power loads on both the PT-415 and the He-10 fridge are quasi-negligible, and are sub-dominant to other loads on these systems (e.g. through wires and mechanical supports).

The MUSIC observing bands are defined using a set of lumped-element bandpass filters placed on microstrips between the phased antenna arrays and the detectors. ${ }^{7}$ By tuning the individual components in the lumpedelement filters we are able to match the pass-bands to the atmospheric observing windows ${ }^{\prime \prime}$ while optimizing the in-band transmission (the average transmission is $\gtrsim 80 \%$ ).

\subsection{IR BLOCKING}

The MUSIC cryostat contains four separate temperature stages, each with a finite amount of cooling power. From warmest to coldest, with maximum acceptable operating temperatures, these stages are: 1) the first stage of the PT-415 ( $\simeq 50 \mathrm{~W}$ at $50 \mathrm{~K}), 2)$ the second stage of the PT-415 ( $1.5 \mathrm{~W}$ at $4 \mathrm{~K}), 3)$ the intermediate cold head of the Chase refrigerator $(\simeq 100 \mu \mathrm{W}$ at $350 \mathrm{mK})$, and 4$)$ the ultracold head of the Chase refrigerator $(\simeq 5 \mu \mathrm{W}$ at $250 \mathrm{mK})$. In general, a filter is required in the optical window of the preceding stage in order to reduce the amount of optical power incident on each temperature stage. Ideally, this filter should reflect all of the radiation that is outside the observing bands and transmit all the radiation within the observing bands. Filters that absorb out of band radiation can also be effective; such filters also radiate out-of-band, but at a colder temperature than the surface they absorb power from.

\footnotetext{
"The 1.33 and $1.02 \mathrm{~mm}$ bands are in a single atmospheric window, these bandpasses were designed to provide roughly
} equal sensitivity to sources with a dusty spectrum. 
In order to determine the optimal filtering scheme for MUSIC we tested a wide range of configurations using absorptive (dielectric) filters. For each configuration we measured the temperature at the center and/or edge of all of the filters, the metal surfaces each filter was clamped to, and each cold stage. Using these data we were able to develop a simple model to robustly predict the temperatures of each filter and temperature stage. To start, the radiative load on each filter can be determined from its emissivity, along with the temperature and emissivity of the adjacent filter. We made the simplifying assumptions that the temperature of the filter is azimuthally symmetric and the emissivity of the filter surface is given by its total absorption, which was calculated using the results in Benford et al. ${ }^{20}$ and Benford et al. ${ }^{21}$ With these assumptions, each annulus receives power via radiation from the adjacent filter and conduction from the adjacent annulus with a higher temperature. Additionally, each annulus loses power via radiation and conduction to the adjacent annulus with a lower temperature. The thermal load on the stage is then the sum of the power radiated from the cold side of the dielectric and the power conducted from the edge of the filter to the clamping surface.

Given this model, the temperature profile of a filter can be determined from a temperature measurement at a single radius, or annulus, if the temperature/emissivity of the previous filter is known. Furthermore, the temperature profile of a filter can also be determined if both the temperature of the surface it is clamped to, along with the thermal conductivity between the filter and the surface, are known. In practice, this simple model predicts temperature profiles that agree with measurements to $\simeq 10 \%$, and power loads on the PT- 415 stages that agree with loadcurve measurements within $\simeq 20 \%$. It should be noted that the thermal conductivity between the dielectric and the clamping surface is relatively poor and is strongly dependent on the amount of clamping force. For example, with a relatively poor clamping design we measured temperature differences $\gtrsim 100 \mathrm{~K}$ between the edge of the dielectric and the clamping surface.

For our final design, a 1 inch thick piece of UHMWPE will be used for the MUSIC cryostat window. This material has excellent transmission at millimeter wavelengths, and is strong enough to maintain vacuum inside the cryostat. Our optics require a window with a $300 \mathrm{~mm}$ diameter clear aperture; in the absence of any filtering the radiation passing through the window will produce $\mathrm{a} \simeq 60 \mathrm{~W}$ load on the first stage of the PT-415. In order to prevent this radiation from reaching the PT- 415 we will have two IR shaders from QMC Instruments behind the window, ${ }^{22}$ the first shader reflects wavelengths below $20 \mu \mathrm{m}$ and the second reflects wavelengths below $100 \mu \mathrm{m}$. Using transmission spectra from QMC, along with the assumption that the filters have a $2 \%$ emissivity, ${ }^{23}$ we estimate that these will reduce the load on the first stage of the PT- 415 to $\simeq 3 \mathrm{~W}$. ${ }^{* *}$

We will use two separate 0.625 inch thick pieces of Teflon to cover the optical opening in the $50 \mathrm{~K}$ radiation shield. These pieces of Teflon will absorb radiation with wavelengths shorter than $\simeq 300 \mu \mathrm{m}$. The center of the first piece of Teflon should reach a temperature of $\simeq 90 \mathrm{~K}$, while the edge is expected to be at a temperature of $\simeq 70 \mathrm{~K}$, and the second piece of Teflon will have a uniform temperature of $\simeq 70 \mathrm{~K}$. Note that the top surface of the $50 \mathrm{~K}$ radiation shield, where these filters will be mounted, is expected to be at $\lesssim 70 \mathrm{~K}$ due to the finite thermal conduction down the sides of the shield. The transmission of this Teflon stack is $>95 \%$ in the $0.86 \mathrm{~mm}$ band, and the optical load transmitted to the second stage of the PT-415 will be a quasi-negligible $\lesssim 100 \mathrm{~mW}$. Additionally, the surfaces of the Teflon will be coated with Zitex ${ }^{21}$ to reduce the in-band reflections.

The optical opening in the $4 \mathrm{~K}$ radiation shield will be covered with a 0.125 inch piece thick of fluorogold sandwiched between two .025 inch thick pieces of Teflon. This sandwich will absorb radiation with wavelengths shorter than $\simeq 500 \mu \mathrm{m}$, and we expect it to be at a uniform temperature of $\lesssim 10 \mathrm{~K}$. As with the previous filter stack, the transmission in-band is excellent, $>93 \%$ in the $0.86 \mathrm{~mm}$ band. Additionally, we will install a $13.5 \mathrm{icm}$ metal mesh filter from QMC inside the $4 \mathrm{~K}$ radiation shield at the Lyot stop. This filter is required because the load on the focal plane, and hence the ultracold head of the Chase refrigerator, without the metal mesh filter is unacceptably large $(\lesssim 5 \mu \mathrm{W})$. Including the metal mesh filter, which reflects radiation with wavelengths below $\simeq 800 \mu \mathrm{m}$, reduces the load on the focal plane to $\lesssim 1 \mu \mathrm{W}$. It is not possible to reproduce the performance of the metal mesh filter using dielectrics; reducing the focal plane load to a similar level using dielectrics significantly

${ }^{* *}$ Note that we have also tested the system using $6 \mathrm{~cm}$ of Zotefoam behind the window; the Zotefoam absorbs radiation with wavelengths below $\simeq 500 \mu \mathrm{m}$ and has a very low thermal conductivity which allows the inner surface to radiatively cool to $\simeq 175 \mathrm{~K}$. With Zotefoam the load on the first stage of the PT-415 is $\simeq 5 \mathrm{~W}$, but it absorbs a non-negligible amount of in-band radiation ( $25 \%$ in the $0.86 \mathrm{~mm}$ band). Therefore, our final design includes the QMC IR shaders rather than Zotefoam. 


\begin{tabular}{|c|cc|cc|}
\hline stage & optical filter(s) & transmission & optical load & cooling power \\
\hline & 1.0 inch UHMWPE & $>99 \%$ & & \\
vac shell $(300 \mathrm{~K})$ & $20 \mu \mathrm{m}$ and $100 \mu \mathrm{m}$ IR shader & $>99 \%$ & $\mathrm{~N} / \mathrm{A}$ & $\mathrm{N} / \mathrm{A}$ \\
\hline PT-415 stage 1 $(50 \mathrm{~K})$ & $\mathrm{x} 20.625$ inch PTFE & $>95 \%$ & $3 \mathrm{~W}$ & $50 \mathrm{~W}$ \\
\hline & $0.25 / 0.125 / 0.25$ PTFE/f-gold/PTFE & $>93 \%$ & & \\
PT-415 stage $2(4 \mathrm{~K})$ & metal mesh $13.5 \mathrm{icm}$ & $>95 \%$ & $\lesssim 100 \mathrm{~mW}$ & $1.5 \mathrm{~W}$ \\
\hline Chase UC $(250 \mathrm{mK})$ & $\mathrm{N} / \mathrm{A}$ & $\mathrm{N} / \mathrm{A}$ & $\lesssim 1 \mu \mathrm{W}$ & $5 \mu \mathrm{W}$ \\
\hline
\end{tabular}

Table 4. The in-band transmission of the optical filter stack at each temperature stage. Also shown is the optical load on each stage, along with the total cooling power of the stage for the maximum acceptable operating temperature.

reduces the transmission in-band due to the relatively gentle cut-off of the dielectrics. A summary of the power load on each temperature stage, along with the in-band transmission of each filter stack, is given in Table 4 .

\subsection{BAND-DEFINING FILTERS}

The phased antenna arrays efficiently absorb radiation over a fairly large bandwidth from $125-375 \mathrm{GHz} ;^{1}$ our relatively narrow observing bands fall within this range and are defined using lumped element filters placed on superconducting feedlines between the antenna arrays and the detectors. For a single pixel, the feedline from the antenna is split into four feedlines which go to the four filters that define each of the observing bands. See Figure 6. Since the filters are highly reflective out of band, this splitting results in negligible power loss. Schematically, the filters are derived from a simple 3-pole Butterworth low-pass filter prototype. A schematic is given in Figure 6. Initially, the capacitance and inductance values are are optimized to give: 1) maximum effective bandwidth (i.e., the integrated bandpass), 2) negligible transmission on or near the main atmospheric absorption lines, and 3) approximately equal sensitivity to dusty objects in the 1.33 and $1.02 \mathrm{~mm}$ bands. Next, the scattering parameters of the feed network and transmission lines between the capacitors and inductors are included in the calculation. Before the circuit model is transferred into a layout for fabrication, each element is optimized using Sonnet to match the design value after accounting for the finite surface impedance of the superconductor; the feed network is further optimized to match the expected power load on each filter. Finally, the filters are then fabricated using superconducting $\mathrm{Nb}$ as the conducting layer and $\mathrm{SiO} 2$ as a dielectric material (dielectric constant of 4 ).

We have fabricated a test focal plane that contains the 1.33, 1.02, and $0.86 \mathrm{~mm}$ observing bands, and measured the bandpasses using a Fourier Transform Spectrometer (FTS). The FTS data agree well with the predicted bandpasses, although the $1.33 \mathrm{~mm}$ band shows some deviations. See Figure 6 . This test data will be used to further optimize the filter designs, and a device with the $2.00 \mathrm{~mm}$ band will be fabricated in the near future.

\section{SUMMARY}

In this manuscript we have presented the optical design for MUSIC, a new (sub)mm camera we are developing for the CSO. The design contains only two powered optics, an off-axis elliptical mirror and a cold refractive lens, and it will provide MUSIC with diffraction limited performance over the maximum FOV possible at the CSO. A cold $(4 \mathrm{~K})$ Lyot stop will be used to control the primary illumination pattern to maximize MUSIC's point source sensitivity while keeping spillover below 1\%. Each pixel on the MUSIC focal plane will be defined using phased antenna arrays, ${ }^{1,2}$ and will be coupled to four separate detectors via four different lumped-element bandpass filters. To minimize the radiative load on the cold stages within the MUSIC cryostat we have used a series of metal-mesh low pass filters and dielectrics; the resulting optical power loads are quasi-negligible compared to the cooling power available at each stage. As we integrate the MUSIC camera several of the optical subsystems have been tested, and perform close to expectations; most of the remaining optical components are in fabrication and will be tested in the near future. 

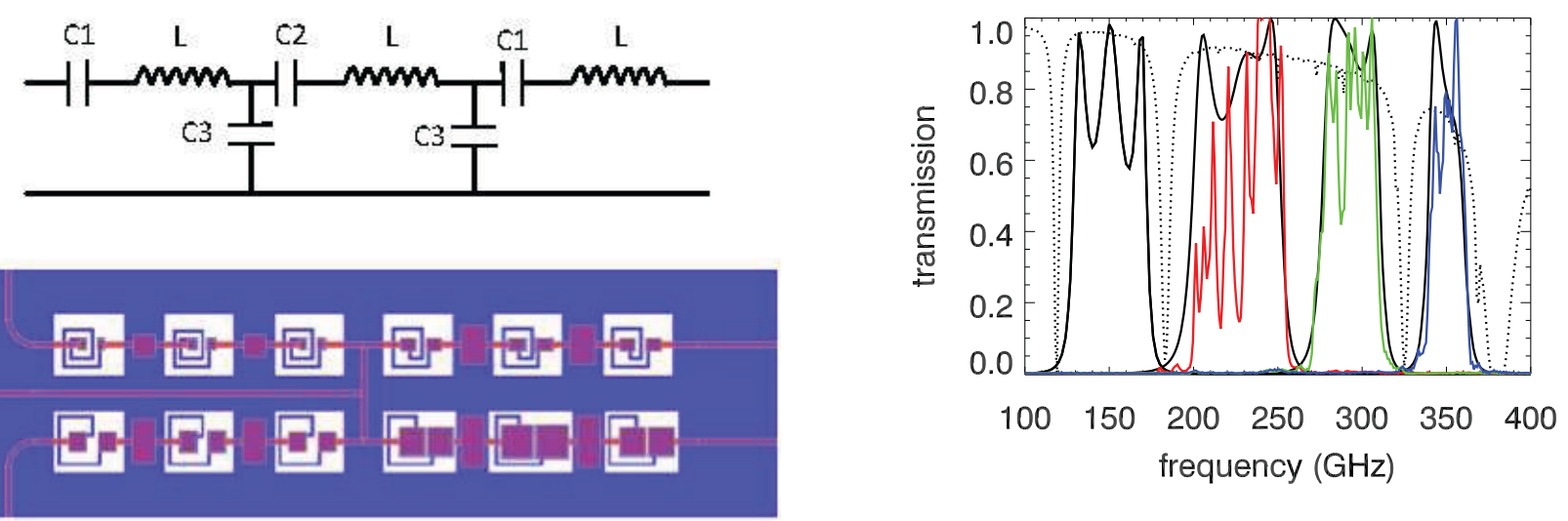

Figure 6. Left top: simple schematic of the bandpass filter design. Left bottom: filter layout. The feedline from the antenna comes in from the left and is split to four bandpass filters. Right: predicted (solid black) bandpasses and measured (solid red, green, and blue) bandpasses overlaid on an atmospheric transmission plot for the CSO with the median $1.68 \mathrm{~mm}$ of precipitable water vapor. ${ }^{24}$

\section{ACKNOWLEDGMENTS}

We acknowledge the assistance of: Sean Lin and Jaime Luna of NASA's Jet Propulsion Laboratory, who engineered the relay optics for the CSO, Marton Precision Manufacturing, Inc. for building the relay optics, Marc Runyan and Randol Aikon for insights from their work developing optical components for SPIDER and BICEP II, Diana Bisel, Kathy Deniston, and Barbara Wertz for exceptional administrative assistance, and the Hilo-based staff of the CSO for help with the design and ongoing installation of the new relay optics. The MKID Camera project is supported by NSF grant AST-0705157 to the University of Colorado, NASA grant NNGC06C71G to Caltech, the Gordon and Betty Moore Foundation, NASA grant NNX10AC83G, and the JPL Research and Technology Development Fund. We are grateful to the Xilinx corporation for their generous donation of the FPGAs needed for the readout electronics. Jack Sayers was supported by a NASA Postdoctoral Program Fellowship and James Schlaerth and Nicole Czakon were partially supported by NASA Graduate Student Researchers Program Fellowships. The research described in this paper was carried out at the Jet Propulsion Laboratory, California Institute of Technology, under a contract with the National Aeronautics and Space Administration.

\section{REFERENCES}

[1] Day, P. K., Czakon, N. G., Day, P. K., Downes, T. P., Duan, R., Gao, J., Glenn, J., Golwala, S., Hollister, M. I., LeDuc, H. G., Maloney, P. R., Mazin, B., Noroozian, O., Nguyen, H. T., Sayers, J., Schlaerth, J., Siegel, S., Vaillancourt, J. E., Vayonakis, A., Wilson, P., and Zmuidzinas, J., "A slot array antenna for a millimeter/submillimeter-wave focal plane," in [Proc. SPIE], 7741-80 (2010).

[2] Kuo, C. L., Bock, J. J., Bonetti, J. A., Brevik, J., Chattopadhyay, G., Day, P. K., Golwala, S., Kenyon, M., Lange, A. E., LeDuc, H. G., Nguyen, H., Ogburn, R. W., Orlando, A., Transgrud, A., Turner, A., Wang, G., and Zmuidzinas, J., "Antenna-coupled TES bolometer arrays for CMB polarimetry," in [Society of PhotoOptical Instrumentation Engineers (SPIE) Conference Series], Presented at the Society of Photo-Optical Instrumentation Engineers (SPIE) Conference 7020 (Aug. 2008).

[3] Maloney, P. R., Czakon, N. G., Day, P. K., Duan, R., Gao, J., Glenn, J., Golwala, S., Hollister, M. I., LeDuc, H. G., Mazin, B., Noroozian, O., Nguyen, H. T., Sayers, J., Schlaerth, J., Vaillancourt, J. E., Vayonakis, A., Wilson, P., and Zmuidzinas, J., "The mkid camera," in [The Thirteenth International Workshop on Low Temperature Detectors - LTD13], Young, B., Cabrera, B., and Miller, A., eds., 172-175 (Nov. 2009).

[4] Maloney, P. R., Czakon, N. G., Day, P. K., Downes, T. P., Duan, R., Gao, J., Glenn, J., Golwala, S., Hollister, M. I., LeDuc, H. G., Mazin, B., Noroozian, O., Nguyen, H. T., Sayers, J., Schlaerth, J., Siegel, S., Vaillancourt, J. E., Vayonakis, A., Wilson, P., and Zmuidzinas, J., "Music for sub/millimeter astrophysics," in [Proc. SPIE], 7741-15 (2010). 
[5] Glenn, J., Bock, J. J., Chattopadhyay, G., Edgington, S. F., Lange, A. E., Zmuidzinas, J., Mauskopf, P. D., Rownd, B., Yuen, L., and Ade, P. A., "Bolocam: a millimeter-wave bolometric camera," in [Advanced Technology MMW, Radio, and Terahertz Telescopes. T. G. Phillips, Ed.], 3357, 326-334, Presented at the Society of Photo-Optical Instrumentation Engineers (SPIE) Conference (July 1998).

[6] Day, P. K., LeDuc, H. G., Mazin, B. A., Vayonakis, A., and Zmuidzinas, J., "A broadband superconducting detector suitable for use in large arrays," Nature 425, 817-821 (2003).

[7] Kumar, S., Submillimeter wave camera using a novel photon detector technology, PhD thesis, Caltech (2007).

[8] Blain, A. W., Smail, I., Ivison, R. J., Kneib, J.-P., and Frayer, D. T., "Submillimeter galaxies," Physics Reports 369, 111-176 (Oct. 2002).

[9] Sunyaev, R. A. and Zeldovich, Y. B., "The Observations of Relic Radiation as a Test of the Nature of X-Ray Radiation from the Clusters of Galaxies," Comments on Astrophysics and Space Physics 4, 173 (Nov. 1972).

[10] Bock, J. J., Glenn, J., Grannan, S. M., Irwin, K. D., Lange, A. E., Leduc, H. G., and Turner, A. D., "Silicon nitride micromesh bolometer arrays for SPIRE," in [Society of Photo-Optical Instrumentation Engineers (SPIE) Conference Series], T. G. Phillips, ed., Society of Photo-Optical Instrumentation Engineers (SPIE) Conference Series 3357, 297-304 (July 1998).

[11] Gear, W. K. and Cunningham, C. R., "SCUBA: A submillimetre camera," in [Liege International Astrophysical Colloquia], B. Kaldeich, ed., Liege International Astrophysical Colloquia 29, 353-358 (Dec. 1990).

[12] Serabyn, E., "A wide-field relay optics system for the Caltech Submillimeter Observatory.," International Journal of Infrared and Millimeter Waves 18, 273-284 (Feb. 1997).

[13] Hollister, M. T., Czakon, N. G., Day, P. K., Downes, T. P., Duan, R., Gao, J., Glenn, J., Golwala, S., LeDuc, H. G., Maloney, P. R., Mazin, B., Noroozian, O., Nguyen, H. T., Sayers, J., Schlaerth, J., Siegel, S., Vaillancourt, J. E., Vayonakis, A., Wilson, P., and Zmuidzinas, J., "Music for sub/millimeter astrophysics," in [Proc. SPIE], 7741-15 (2010).

[14] Sayers, J., Golwala, S., and et al., "Expected performance of a large-format multi-color (sub)millimeter camera at the caltech submillimeter observatory,".

[15] Sayers, J., Golwala, S. R., Rossinot, P., Ade, P. A. R., Aguirre, J. E., Bock, J. J., Edgington, S. F., Glenn, J., Goldin, A., Haig, D., Lange, A. E., Laurent, G. T., Mauskopf, P. D., and Nguyen, H. T., "A Search for Cosmic Microwave Background Anisotropies on Arcminute Scales with Bolocam," The Astrophysical Journal 690, 1597-1620 (Jan. 2009).

[16] Bock, J. J., Rocket-borne observation of singly ionized carbon $158 \mathrm{~m}$ emission from the diffuse interstellar medium, $\mathrm{PhD}$ thesis, University of California, Berkeley (1994).

[17] Schlaerth, J., Czakon, N. G., Day, P. K., Downes, T. P., Duan, R., Gao, J., Glenn, J., Golwala, S., Hollister, M. I., LeDuc, H. G., Maloney, P. R., Mazin, B., Noroozian, O., Nguyen, H. T., Sayers, J., Siegel, S., Vaillancourt, J. E., Vayonakis, A., Wilson, P., and Zmuidzinas, J., "Mkid multicolor array status and results from democam," in [Proc. SPIE], 7741-8 (2010).

[18] Bhatia, R. S., Chase, S. T., Edgington, S. F., Glenn, J., Jones, W. C., Lange, A. E., Maffei, B., Mainzer, A. K., Mauskopf, P. D., Philhour, B. J., and Rownd, B. K., "A three-stage helium sorption refrigerator for cooling of infrared detectors to $280 \mathrm{mK}, "$ Cryogenics 40, 685-691 (2000).

[19] Bhatia, R. S., Chase, S. T., Jones, W. C., Keating, B. G., Lange, A. E., Mason, P. V., Philhour, B. J., and Sirbi, G., "Closed cycle cooling of infrared detectors to $250 \mathrm{mK}$," Cryogenics 42, 113-122 (2002).

[20] Benford, D. J., Kooi, J. W., and Serabyn, E., "Spectroscopic Measurements of Optical Components Around 1 Terhertz," ISSTT Proceedings , 405-413 (1998).

[21] Benford, D. J., Gaidis, M. C., and Kooi, J. W., "Optical Properties of Zitex in the Infrared to Submillimeter," Applied Optics 42, 5118-5122 (2003).

[22] Ade, P. A. R., Pisano, G., Tucker, C., and Weaver, S., "A review of metal mesh filters," in [Millimeter and Submillimeter Detectors and Instrumentation for Astronomy III. J. Zmuidzinas, W. S. Holland, S. Withington, and W. D. Duncan, Eds. Proceedings of the SPIE.], 6275, 62750U, Presented at the Society of Photo-Optical Instrumentation Engineers (SPIE) Conference (July 2006).

[23] Hollister, M. I. personal communication (2010).

[24] Pardo, J. R., Cernicharo, J., and Serabyn, E., "Atmospheric transmission at microwaves (ATM): an improved model for millimeter/submillimeter applications," IEEE Transactions on Antennas and Propagation 49, 1683-1694 (Dec. 2001). 